\title{
Charmed baryons in nuclear matter
}

\author{
T. F. Caramés, ${ }^{1, *}$ C. E. Fontoura, ${ }^{2, \dagger}$ G. Krein, ${ }^{3, \Downarrow}$ J. Vijande, ${ }^{4, \S}$ and A. Valcarce ${ }^{1, \|}$ \\ ${ }^{1}$ Departamento de Física Fundamental e IUFFyM, Universidad de Salamanca, \\ E-37008 Salamanca, Spain \\ ${ }^{2}$ Instituto Tecnológico de Aeronáutica, DCTA, 12228-900 São José dos Campos, SP, Brazil \\ ${ }^{3}$ Instituto de Física Teórica, Universidade Estadual Paulista, Rua Dr. Bento Teobaldo Ferraz, 271 - Bloco II, \\ 01140-070 São Paulo, SP, Brazil \\ ${ }^{4}$ Unidad Mixta de Investigación en Radiofísica e Instrumentación Nuclear en Medicina (IRIMED), \\ Instituto de Investigación Sanitaria La Fe (IIS-La Fe)-Universitat de Valencia (UV) and IFIC (UV-CSIC), \\ E-46100 Valencia, Spain
}

(Received 31 October 2018; published 18 December 2018)

\begin{abstract}
We study the temperature and baryon density dependence of the masses of the lightest charmed baryons $\Lambda_{c}, \Sigma_{c}$ and $\Sigma_{c}^{*}$. We also look at the effects of the temperature and baryon density on the binding energies of the $\Lambda_{c} N$ and $\Lambda_{c} \Lambda_{c}$ systems. Baryon masses and baryon-baryon interactions are evaluated within a chiral constituent quark model. Medium effects are incorporated in those parameters of the model related to the dynamical breaking of chiral symmetry, which are the masses of the constituent quarks, the $\sigma$ and $\pi$ meson masses, and quark-meson couplings. We find that while the in-medium $\Lambda_{c}$ mass decreases monotonically with temperature, those of $\Sigma_{c}$ and $\Sigma_{c}^{*}$ have a nonmonotonic dependence. These features can be understood in terms of a simple group theory analysis regarding the one-gluon exchange interaction in those hadrons. The in-medium $\Lambda_{c} N$ and $\Lambda_{c} \Lambda_{c}$ interactions are governed by a delicate balance involving a stronger attraction due to the decrease of the $\sigma$ meson mass, suppression of coupled-channel effects and lower thresholds, leading to shallow bound states with binding energies of a few MeV. The $\Lambda_{c}$ baryon could possibly be bound to a large nucleus, in qualitative agreement with results based on relativistic mean field models or QCD sum rules. Ongoing experiments at RHIC or LHCb or the planned ones at FAIR and J-PARC may take advantage of the present results.
\end{abstract}

DOI: 10.1103/PhysRevD.98.114019

\section{INTRODUCTION}

The study of hadrons containing charm quarks is of broad interest nowadays in our quest to understand the fundamental theory of the strong interaction, quantum chromodynamics (QCD). Spectroscopy of such hadrons, which has been at the forefront of research since the discovery of the charm quark in 1974, continues receiving most of the attention in light of the continuous discovery during the last decade of the so-called $X Y Z$ exotic mesons, most of which have been observed above the charm production threshold. Their internal structure is still under

\footnotetext{
*carames@usal.es

†ce.fontoura@unesp.br

gastao.krein@unesp.br

javier.vijande@uv.es

valcarce@usal.es
}

Published by the American Physical Society under the terms of the Creative Commons Attribution 4.0 International license. Further distribution of this work must maintain attribution to the author(s) and the published article's title, journal citation, and DOI. Funded by SCOAP. scrutiny, the main conjectures being threshold effects, molecular structures or compact multiquark states [1-7]. The major difficulty here, as in any other instance involving hadron spectroscopy, is our poor understanding of the low energy regime of $\mathrm{QCD}$, which is dominated by nonperturbative phenomena like color confinement and mass generation. In this respect, the study of charmed hadrons in matter offers an extremely promising possibility for exploring such phenomena. In a medium composed predominantly by light quarks, like an atomic nucleus whose properties are determined by the nonperturbative physics at the energy scale $\Lambda_{\mathrm{QCD}}$, the charm quark plays the role of an impurity particle because of its large mass, $m_{c} \simeq$ $5 \Lambda_{\mathrm{QCD}}$ [8]. This is to say that the vacuum properties of a charm quark are little or not at all modified in an atomic nucleus and any change in the properties of a hadron containing charm can be linked to its light constituents. Indeed, the proposal made long time ago of using charmonia as probes of the properties of the excited matter produced in a relativistic heavy ion collision is a prime example of such a possibility [9]. Other possibilities 
include $D$-mesic nuclei, charmed hypernuclei and nuclearbound charmonia [10,11].

In recent years, there has been an impressive experimental progress in the spectroscopy of heavy baryons, mainly in the charm sector. The LHCb Collaboration at the Large Hadron Collider (LHC), in particular, is engaged in an extensive program aimed at analyses of charmed hadrons produced in the environment of high-energy proton-proton collisions [12] and has already reported the observation of five new narrow excited $\Omega_{c}$ states [13]. Also, coalescence and statistical hadronization models [14] predict that not only charmed baryons $Y_{c}=\left(\Lambda_{c}, \Sigma_{c}, \Xi_{c}, \Omega_{c}, \cdots\right)$, but also $Y_{c} N$ bound or resonant states, where $N=(p, n)$, are produced at relatively high rates in the environment of a heavy-ion collision at the LHC. In addition, in the coming years experiments aimed at producing charmed hypernuclei, in which a $Y_{c}$ baryon is bound to a nucleus, are becoming realistic at the planned installation of a $50 \mathrm{GeV}$ high-intensity proton beam at Japan Proton Accelerator Research Complex (J-PARC) $[15,16]$. There are also planned experiments by the $\bar{P} A N D A$ Collaboration at the Facility for Antiproton Ion Research (FAIR) $[17,18]$ to produce charmed hadrons by annihilating antiprotons on nuclei.

These experimental prospects have reinvigorated studies of the low-energy $Y_{c} N$ interactions in free space and also in matter [19-40]. More recently, also $Y_{c} Y_{c}$ interactions are becoming focus of interest [41-49]. The complete lack of experimental information on the elementary $Y_{c} N$ and $Y_{c} Y_{c}$ interactions in free space imposes great difficulties in accessing in-medium effects.

Although recent results from lattice simulations of the $Y_{c} N$ interactions provide important guidance [36,37], they are still obtained with unphysical pion masses and need to be extrapolated using, e.g., a chiral effective field theory [31]. In order to make progress, the strategies used so far rely on the use of models constrained as much as possible by symmetry arguments, analogies with similar systems, and the use of different degrees of freedom.

Relativistic mean field models, based either on quark degrees of freedom [50] or hadronic degrees of freedom [51] have been widely used to study medium dependence of the strange hyperons $Y=(\Lambda, \Sigma, \cdots)$ and are natural candidates to be extended to the charm sector. Calculations using quark degrees of freedom [21,22] predict that some of the $Y_{c}$ baryons are likely to be bound to sufficiently large nuclei. A similar calculation [35], but based on a nonlinear meson-exchange mean field model, predicts that $\Lambda_{c}$ can be bound to nuclei as small as ${ }^{12} \mathrm{C}$.

QCD sum rules is another technique that has been used to investigate properties of charmed baryons in nuclear matter, sometimes with contradictory conclusions. One major source of uncertainty with the QCD sum rules is the lack of reliable information on the in-medium condensates, i.e., the thermodynamical average of products of quark and gluon operators. Although the lowest-dimension in-medium condensates are relatively well constrained by chiral symmetry and the trace anomaly, condensates of higher dimension are commonly treated in the so-called factorization hypothesis, in that they are written as products of the lowest-dimension condensates [52]. For example, using the factorization hypothesis Ref. [24] finds that the mass of the $\Lambda_{c}$ increases in nuclear matter, which means that $\Lambda_{c}$ feels an average repulsive potential. The same author concludes in Ref. [25] that the mass of the $\Sigma_{c}$ baryons decreases, while Ref. [38] finds the opposite result; both calculations use the factorization hypothesis. Still, Ref. [32], also using the factorization hypothesis, finds that the mass of $\Lambda_{c}$ decreases considerably in nuclear matter. Ref. [33] examines critically the role played by the factorization hypothesis and finds, in particular, that the results based on QCD sum rules depend strongly on the density dependence of the four-quark condensate. When using a density dependence based on a factorization hypothesis for the four-quark condensate, that reference predicts that the $\Lambda_{c}$ mass increases with density, while the opposite behavior is obtained when using a density dependence predicted by a perturbative chiral quark model for the four-quark condensate. The latter result points toward the possibility that the $\Lambda_{c}$ might be bound to a nucleus, provided, of course, it can be produced almost at rest in the nucleus. The authors of Ref. [33] still perform a similar analysis for the $\Lambda$ hyperon and find that when the factorized four-quark condensate is employed, a very strong repulsion is obtained and thereof favors the unfactorized four-quark condensate, which predicts a weak attraction, in qualitative agreement with the mass shift of $\Lambda$ in nuclear matter as extracted from the binding energies of hypernuclei.

Clearly, these studies reveal that our knowledge on in-medium mass of the $\Lambda_{c}$ is still very rudimentary and suggest the need of further consideration. As already mentioned, our ability of making first-principles, analytical calculations of nonperturbative QCD phenomena is very limited and, thus, the use of models is still a valid alternative for making progress. Within such a perspective, in the present work we employ a widely used chiral constituent quark model $[53,54]$ to evaluate the in-medium masses of charmed hadrons as well as their in-medium lowenergy interactions with nucleons and other charmed baryons. The model provides a very good description of the low-lying spectrum of the light and charmed hadrons $[55,56]$. The vacuum values of the parameters of the model, which are the masses of constituent quarks, the $\pi$ and $\sigma$ meson masses and their coupling constants to the light constituent quarks are therefore well constrained. To evaluate the temperature and baryon density dependence of those parameters we employ the Nambu-Jona-Lasinio (NJL) model [57,58], following the strategy set up in our 
previous work in Ref. [59] on the in-medium properties of a $\Delta \bar{D}^{*}$ molecule.

The paper is organized as follows. In Sec. II we outline the basic ingredients of the chiral constituent quark model used for the study of the one- and two-baryon problems. In Sec. III we present and discuss the results for the inmedium masses of the charmed baryons $Y_{c}=\left(\Lambda_{c}, \Sigma_{c}, \Sigma_{c}^{*}\right)$. In Sec. IV we present numerical results for the in-medium $\Lambda_{c} N$ and $\Lambda_{c} \Lambda_{c}$ interactions in comparison to other approaches in the literature. Finally, in Sec. V we summarize the main conclusions of our work.

\section{QUARK-QUARK AND BARYON-BARYON INTERACTIONS}

In this section we define the chiral constituent quark model used in the present work [53]. The model was proposed in the early $1990 \mathrm{~s}$ in an attempt to obtain a simultaneous description of the light baryon spectrum and the nucleon-nucleon interaction. It was later on generalized to all flavor sectors [54]. In this model, hadrons are described as clusters of three interacting massive (constituent) quarks. The masses of the quarks are generated by the dynamical breaking of the original $S U(2)_{L} \otimes S U(2)_{R}$ chiral symmetry of the QCD Lagrangian at a momentum scale of the order of $\Lambda_{\mathrm{CSB}}=4 \pi f_{\pi} \sim 1 \mathrm{GeV}$, where $f_{\pi}$ is the pion electroweak decay constant. For momenta typically below that scale, when using the linear realization of chiral symmetry, light quarks interact through potentials generated by the exchange of pseudoscalar Goldstone bosons $(\pi)$ and their chiral partner $(\sigma)$ :

$$
V_{\chi}\left(\vec{r}_{i j}\right)=V_{\sigma}\left(\vec{r}_{i j}\right)+V_{\pi}\left(\vec{r}_{i j}\right)
$$

where

$$
\begin{aligned}
V_{\sigma}\left(\vec{r}_{i j}\right)= & -\frac{g_{\mathrm{ch}}^{2}}{4 \pi} \frac{\Lambda^{2}}{\Lambda^{2}-m_{\sigma}^{2}} m_{\sigma}\left[Y\left(m_{\sigma} r_{i j}\right)-\frac{\Lambda}{m_{\sigma}} Y\left(\Lambda r_{i j}\right)\right], \\
V_{\pi}\left(\vec{r}_{i j}\right)= & \frac{g_{\mathrm{ch}}^{2}}{4 \pi} \frac{m_{\pi}^{2}}{12 M_{i} M_{j}} \frac{\Lambda^{2}}{\Lambda^{2}-m_{\pi}^{2}} m_{\pi} \\
& \times\left\{\left[Y\left(m_{\pi} r_{i j}\right)-\frac{\Lambda^{3}}{m_{\pi}^{3}} Y\left(\Lambda r_{i j}\right)\right] \vec{\sigma}_{i} \cdot \vec{\sigma}_{j}\right. \\
& \left.+\left[H\left(m_{\pi} r_{i j}\right)-\frac{\Lambda^{3}}{m_{\pi}^{3}} H\left(\Lambda r_{i j}\right)\right] S_{i j}\right\}\left(\vec{\tau}_{i} \cdot \vec{\tau}_{j}\right) .
\end{aligned}
$$

$g_{\mathrm{ch}}^{2} / 4 \pi$ is the chiral coupling constant, $M_{i}=\left(M_{u}, M_{d}\right)$ are the masses of the constituent quarks, $\Lambda \sim \Lambda_{\mathrm{CSB}}, Y(x)$ is the standard Yukawa function defined by $Y(x)=e^{-x} / x$, $H(x)=\left(1+3 / x+3 / x^{2}\right) Y(x)$, and $S_{i j}=3\left(\vec{\sigma}_{i} \cdot \hat{r}_{i j}\right)\left(\vec{\sigma}_{j}\right.$. $\left.\hat{r}_{i j}\right)-\vec{\sigma}_{i} \cdot \vec{\sigma}_{j}$ is the quark tensor operator.

Perturbative QCD effects are taken into account through the one-gluon-exchange (OGE) potential [60]:

$$
\begin{aligned}
V_{\mathrm{OGE}}\left(\vec{r}_{i j}\right)= & \frac{\alpha_{s}}{4} \vec{\lambda}_{i}^{\mathrm{c}} \cdot \vec{\lambda}_{j}^{\mathrm{c}}\left[\frac{1}{r_{i j}}-\frac{1}{4}\left(\frac{1}{2 M_{i}^{2}}+\frac{1}{2 M_{j}^{2}}+\frac{2 \vec{\sigma}_{i} \cdot \vec{\sigma}_{j}}{3 M_{i} M_{j}}\right)\right. \\
& \left.\times \frac{e^{-r_{i j} / r_{0}}}{r_{0}^{2} r_{i j}}-\frac{3 S_{i j}}{4 M_{i} M_{j} r_{i j}^{3}}\right],
\end{aligned}
$$

where $\lambda^{c}$ are the $S U(3)$ color matrices, $r_{0}=\hat{r}_{0} / \nu$ is a flavor-dependent regularization scaling with the reduced mass $\nu$ of the interacting pair, and $\alpha_{s}$ is the scale-dependent strong coupling constant given by [54],

$$
\alpha_{s}(\nu)=\frac{\alpha_{0}}{\ln \left[\left(\nu^{2}+\mu_{0}^{2}\right) / \gamma_{0}^{2}\right]},
$$

where $\alpha_{0}=2.118, \mu_{0}=36.976 \mathrm{MeV}$ and $\gamma_{0}=0.113 \mathrm{fm}^{-1}$. This equation gives rise to $\alpha_{s} \sim 0.54$ for the light-quark sector, $\alpha_{s} \sim 0.43$ for $u c$ pairs, and $\alpha_{s} \sim 0.29$ for $c c$ pairs.

Finally, any model imitating QCD should incorporate confinement. Although it is a very important term from the spectroscopic point of view, it is negligible for the hadronhadron interaction. Lattice calculations suggest a screening effect on the potential when increasing the interquark distance [61] which is modeled here by,

$$
V_{\mathrm{CON}}\left(\vec{r}_{i j}\right)=-a_{c}\left(1-e^{-\mu_{c} r_{i j}}\right)\left(\vec{\lambda}_{i} \cdot \vec{\lambda}^{c}{ }_{j}\right),
$$

where $a_{c}$ and $\mu_{c}$ are the strength and range parameters. Once perturbative (one-gluon exchange) and nonperturbative (confinement and dynamical chiral symmetry breaking) aspects of QCD have been incorporated, one ends up with a quark-quark interaction of the form,

$$
\begin{aligned}
& V_{q_{i} q_{j}}\left(\vec{r}_{i j}\right) \\
& =\left\{\begin{array}{l}
{\left[q_{i} q_{j}=n n\right] \Rightarrow V_{\mathrm{CON}}\left(\vec{r}_{i j}\right)+V_{\mathrm{OGE}}\left(\vec{r}_{i j}\right)+V_{\chi}\left(\vec{r}_{i j}\right)} \\
{\left[q_{i} q_{j}=c n / c c\right] \Rightarrow V_{\mathrm{CON}}\left(\vec{r}_{i j}\right)+V_{\mathrm{OGE}}\left(\vec{r}_{i j}\right)}
\end{array},\right.
\end{aligned}
$$

where $n$ stands for the light quarks $u$ and $d$. Notice that for the particular case of heavy quarks ( $c$ or $b$ ) chiral symmetry is explicitly broken and therefore boson exchanges associated with the dynamical breaking of chiral symmetry do not contribute. The parameters of the model are the same that have been used for the study of the one- and twobaryon systems in vacuum, and for completeness are quoted in Table I.

TABLE I. Quark-model parameters.

\begin{tabular}{lclc}
\hline \hline$M_{u, d}(\mathrm{MeV})$ & 313 & $m_{\pi}\left(\mathrm{fm}^{-1}\right)$ & 0.70 \\
$m_{c}(\mathrm{MeV})$ & 1752 & $\Lambda\left(\mathrm{fm}^{-1}\right)$ & 4.2 \\
$\hat{r}_{0}(\mathrm{MeV} \mathrm{fm})$ & 28.170 & $a_{c}(\mathrm{MeV})$ & 230 \\
$\mu_{c}\left(\mathrm{fm}^{-1}\right)$ & 0.70 & & \\
$g_{\mathrm{ch}}^{2} /(4 \pi)$ & 0.54 & & \\
$m_{\sigma}\left(\mathrm{fm}^{-1}\right)$ & 3.42 & & \\
\hline \hline
\end{tabular}


In order to derive the $B_{n} B_{m} \rightarrow B_{k} B_{l}$ interaction from the basic $q q$ interaction defined above, we use a BornOppenheimer approximation where the quark coordinates are integrated out keeping $R$ fixed, the resulting interaction being a function of the two-baryon relative distance. A thorough discussion of the model can be found elsewhere $[53,54]$.

\section{IN-MEDIUM CHARMED BARYON MASSES}

Medium effects on the baryon masses and baryonbaryon interactions are incorporated within a quasiparticle picture of the nuclear many-body problem, in which the parameters of the underlying quark model carry the medium effects [59]. This is similar to the calculations of $Y_{c}$ masses in the quark-meson coupling model $[19,21]$, in which meson mean fields sourced by quark scalar and vector in-medium densities couple to current quarks within the baryons, and also in QCD sum rules [24,25,32,33,38], in which medium effects are carried by (quark, gluon and mixed quark-gluon) condensates. In the chiral quark model that we employ here, the temperature, $T$, and baryon density, $\rho_{B}$, dependence of model parameters are those predicted by the NJL model. This choice is motivated by the fact that the bosonized version of the NJL model with $\sigma$ and $\pi$ mesons $[62,63]$ leads to the same Yukawa quarkmeson couplings as those in the chiral constituent quark model discussed in Sec. II. In addition, it gives very simple expressions for the masses and quark-meson couplings in vacuum and also for nonzero $T$ and $\mu$. Moreover, for sufficiently low values of $T$ and $\rho_{B}$, the NJL model reproduces [63-66] the model-independent predictions derived in the context of chiral perturbation theory for the in-medium quark condensate $\langle\langle\bar{q} q\rangle\rangle[52,67,68]$ :

$$
\frac{\langle\langle\bar{q} q\rangle\rangle}{\langle\bar{q} q\rangle} \simeq 1-\frac{T^{2}}{8 f_{\pi}^{2}}-\frac{1}{3} \frac{\rho_{B}}{\rho_{0}},
$$

where $\langle\bar{q} q\rangle$ is the vacuum light quark condensate, $f_{\pi}$ the vacuum pion electroweak decay constant, and $\rho_{0}$ the baryon saturation density of nuclear matter. For $\rho_{B}=0$, this prediction would be valid for $T \lesssim 0.1 \mathrm{GeV}$ [67]. In a treatment that includes thermal excitations of the pions, the NJL model reproduces Eq. (7) very well up to $T \simeq$ $0.1 \mathrm{GeV}$ [66]. When the model is solved in the Hartree approximation, the one used in the present work, it gives a value for $\langle\langle\bar{q} q\rangle\rangle /\langle\bar{q} q\rangle$ that is $10 \%$ larger than given by Eq. (7). In the particular case of $\rho_{B} / \rho_{0}=0.5$, which corresponds to $\mu=0.19 \mathrm{GeV}$, it is $25 \%$ larger. While the pion mass is protected by chiral symmetry, i.e., its mass does not change while chiral symmetry is not restored, the masses of the constituent quarks and of the $\sigma$ meson are to a good approximation proportional to the quark condensate. Despite the existence of these model independent results, a model is still required mainly because further input is needed, namely the $T$ and $\rho_{B}$ dependence of the quark-meson coupling constants. The relevant equations determining the $T$ and $\rho_{B}$ dependence of the masses and couplings are well known since long time [63-66]. The particular implementation of the model is the one used in our previous work in Ref. [59]. We refer the reader to that reference for details and also discussions on the limitations of the calculations.

The in-medium dependence of the charmed baryon masses are readily obtained by solving the bound-state problem of three constituent quarks as detailed in Refs. $[55,56]$ with the temperature, $T$, and baryon chemical potential, $\mu$, dependence of the quark and meson masses and the quark-meson couplings as obtained in Ref. [59]. In Fig. 1 we depict the variation of the masses of $\Lambda_{c}, \Sigma_{c}$, and $\Sigma_{c}^{*}$ baryons as a function of $T$ for different values of the baryon chemical potential $\mu$. As one can see, we find that the mass of the $\Lambda_{c}$ decreases monotonically with both $T$ and $\mu$, in qualitative agreement with the results of Refs. [21,32] and those of Ref. [33] when using the unfactorized four-quark condensate with a density dependence taken from a perturbative chiral quark model. In more quantitative terms, our model predicts a mass decrease of $\Delta M_{\Lambda_{c}}=72 \mathrm{MeV}$ at $\rho_{B} / \rho_{0}=1$, which at the highest value of $\mu$ used, $\mu=0.2 \mathrm{GeV}$, corresponds to $T \simeq 0.12 \mathrm{GeV}$. At this values, $\langle\langle\bar{q} q\rangle\rangle /\langle\bar{q} q\rangle \simeq 0.7$. This mass decrease is smaller than the one obtained by the QMC model [21], $\Delta M_{\Lambda_{c}} \simeq 122 \mathrm{MeV}$, and much larger than those obtained in the QCD sum rules calculation of Refs. [32,33], which give $\Delta M_{\Lambda_{c}} \simeq 10 \mathrm{MeV}$. Although in the QCD sum rules calculations, $\langle\langle\bar{q} q\rangle\rangle /\langle\bar{q} q\rangle$ at $\rho_{B} / \rho_{0}=1$ is essentially equal to the one in our calculation, the results show that there are differences in the way chiral symmetry restoration works in the different models. While in the present calculation the interpretation for the decrease in the mass of $\Lambda_{c}$ can be made (see the discussion in the next paragraph) in terms of the interplay between increased kinetic energy and the spinspin interactions due to gluon and pion exchanges, in the QCD sum rules such an assessment is more difficult, as discussed e.g., in Ref. [33]. In the case of the QMC model, a role similar to the quark condensate is played by a scalar nuclear mean field. The interplay between increased kinetic terms and attractive zero-point and c.m. energies is clearly exposed in the hadron mass formulae [21]. On the other hand, the QCD sum rule conclusions of Refs. [24,25] and those of Ref. [33] based on the factorized four-quark condensate disagree with our predictions. At this point, it needs to be said that Ref. [33] notes how the density dependence of the factorized four-quark condensate is too strong to explain the observed binding of $\Lambda$ in nuclei, a feature which advocates in favor of the unfactorized weak density dependence of the perturbartive chiral quark model. On the other hand, the masses of $\Sigma_{c}$ and $\Sigma_{c}^{*}$ do not decrease monotonically with $T$, they start decreasing for small values of $T$ but then turn over and become larger than their vacuum values for higher values of $T$. The turnover temperature decreases as $\mu$ increases. 

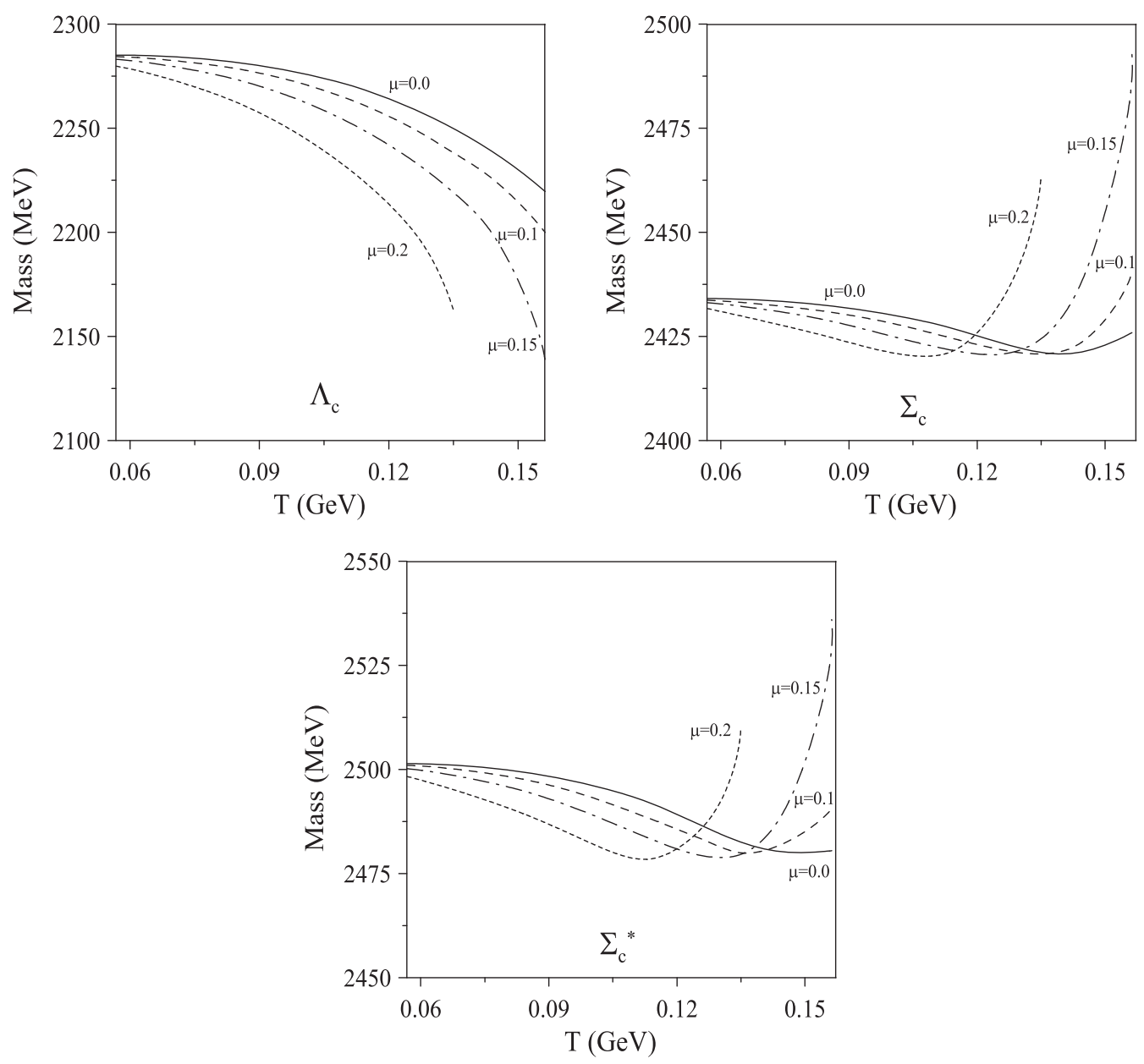

FIG. 1. Masses of $\Lambda_{c}, \Sigma_{c}$ and $\Sigma_{c}^{*}$ baryons as a function of the temperature for the different values of the baryon chemical potential $\mu$ (in $\mathrm{GeV}$ ).

The behavior of the in-medium masses of charmed baryons can be easily understood in terms of a simple group theory analysis [69]. In an approximation in which the heavy-quark masses are taken $M_{Q} \rightarrow \infty$, the angular momentum of the light degrees of freedom is a good quantum number. Thus, heavy-quark baryons belong either to the flavor $S U(3)$ antisymmetric $\overline{\mathbf{3}}_{\mathrm{F}}$ representation, or to the symmetric $\mathbf{6}_{\mathbf{F}}$ representation. The spin of the light diquark is 0 for $\overline{\mathbf{3}}_{\mathbf{F}}$, while it is 1 for $\mathbf{6}_{\mathbf{F}}$. Thus, while the spin of the ground state baryons is $1 / 2$ for the $\overline{\mathbf{3}}_{\mathrm{F}}$ representation, which contains among others the $\Lambda_{c}$ baryon, it can be both $1 / 2$ or $3 / 2$ for the $\mathbf{6}_{\mathbf{F}}$ representation, which contains among others the $\Sigma_{c}$ and the $\Sigma_{c}^{*}$, respectively. Therefore, heavy hadrons would form doublets, in that $\Sigma_{Q}$ and $\Sigma_{Q}^{*}$ would be mass-degenerate in the limit $M_{Q} \rightarrow \infty$ and, away from this limit, there is a mass splitting due to the spin-spin interaction at order $1 / M_{Q}$. The mass difference between states belonging to the flavor $\overline{\mathbf{3}}_{\mathbf{F}}$ and $\mathbf{6}_{\mathbf{F}}$ representations tends to a constant when the heavy quark mass $M_{Q} \rightarrow \infty$, due to the dynamics of the light diquark subsystem, so that:

$$
\begin{aligned}
\mathrm{M}\left[\Sigma_{c}^{*}\right]-\mathrm{M}\left[\Sigma_{c}\right] & \Rightarrow \Delta \mathrm{M}\left(\left[\mathbf{6}_{\mathbf{F}}\right]-\left[\mathbf{6}_{\mathbf{F}}\right]\right) \equiv V_{\text {light-charm }} \\
\mathrm{M}\left[\Sigma_{c}^{*}\right]-\mathrm{M}\left[\Lambda_{c}\right] & \Rightarrow \Delta \mathrm{M}\left(\left[\mathbf{6}_{\mathbf{F}}\right]-\left[\overline{\mathbf{3}}_{\mathbf{F}}\right]\right) \equiv V_{\text {light-light }}
\end{aligned}
$$

Let us note that in $\Lambda_{c}$ there is an attractive $u d$ diquark ("good" diquark) with color $\overline{\mathbf{3}}$, spin 0 and isospin 0 , whereas in $\Sigma_{c}$ and $\Sigma_{c}^{*}$ there is a repulsive $u d$ diquark ("bad" diquark) with color $\overline{\mathbf{3}}$, but spin 1 and isospin 1 . This is why $\Sigma_{c}$ and $\Sigma_{c}^{*}$ baryons follow a similar behavior with temperature and density whereas the $\Lambda_{c}$ has a completely different behavior, its mass diminishing due to the attractive character of the one-gluon exchange for a spin zero diquark, effect that is increased when the mass of the quark diminishes. As can be seen in Eq. (8), the mass difference between the members of the $\overline{\mathbf{3}}_{\mathbf{F}}$ and $\mathbf{6}_{\mathbf{F}}$ comes determined by the dynamics of the two-light quarks. Being the spin-isospin pairs in a symmetric state for both configurations it is the switch of the spin-color pairs symmetry, symmetric for the flavor $\overline{\mathbf{3}}_{\mathbf{F}}$ representation and antisymmetric for the $\mathbf{6}_{\mathbf{F}}$, the responsible for the nonmonotonic behavior of the $\Sigma_{c}$ and $\Sigma_{c}^{*}$ masses. While in the $\Lambda_{c}$ all contributions (except for the kinetic energy) 
are attractive, in the members of the $\mathbf{6}_{\mathbf{F}}$ representation the spin-color interaction becomes repulsive. For small variations of the mass of the light quarks there is a compensation between the attractive character of the pseudo-Goldstone boson exchange interaction and the repulsive OGE and kinetic energy contributions. For larger temperatures, for a given baryon chemical potential, the repulsive character of the spin-color interaction, depending on the regularization of a $\delta$-function through the reduced mass of the interacting quarks [see Eq. (3)], together with the increase of the repulsive kinetic energy, dominates the attractive contributions. For the $\Lambda_{c}$ case, the spin-color interaction is also attractive, increasing in this way the slope of the decrease of the $\Lambda_{c}$ mass when the $\Sigma_{c}$ and $\Sigma_{c}^{*}$ masses present the turnover.

These results show that charmed baryons offer an ideal laboratory for learning on temperature and density effects on the phenomenon of dynamical chiral symmetry breaking. The different two-quark subsystems, heavy-light and light-light, are clearly disentangled by the way they react to changes in the quark masses, which in the case of baryons affects primarily the spin-spin interaction [59].

\section{IN-MEDIUM BINDING OF CHARMED BARYONS}

In this section we investigate how the interaction of the $\Lambda_{c}$ baryon with nucleons and with other charmed baryons is modified in a medium at finite $T$ and $\mu$. We note that our calculation is particularly applicable for a medium similar to the one formed in a high-energy heavy-ion collision, in which quarks coalesce to form weakly bound hadron molecules [70]. At finite temperature and/or baryon chemical potential there are several competing effects. On one hand, the interactions grow due to the decrease in $m_{\sigma}$ at finite $T$ and $\mu$ providing a stronger interaction. Besides, thresholds are modified due to the changes in the masses of the charmed baryons, shown in Fig. 1, and the nucleon, see Fig. 2(b) of Ref. [59]. Finally, coupled-channel effects through the $\Lambda_{c} \leftrightarrow \Sigma_{c}$ conversion are less important because, as seen in the previous section, the mass difference increases both with the temperature and the baryon chemical potential. In the following, we investigate the $T$ and $\mu$ dependence of the $\Lambda_{c} N$ and $\Lambda_{c} \Lambda_{c}$ interactions taking into account all those effects. We note that in the present model these two-baryon systems are not bound in vacuum $[29,46]$.

To study the possible existence of two-baryon bound states, we solve the Lippmann-Schwinger equation for negative energies as has been detailed in Ref. [59] and examine the Fredholm determinant $D_{F}(E)$ at zero energy [71]. For noninteracting systems $D_{F}(0)=1$, for an attractive two-baryon interaction $0<D_{F}(0)<1$, and when there exits a bound state $D_{F}(0)<0$. Making use of the in-medium baryon masses and baryon-baryon interactions obtained with the chiral constituent quark model with

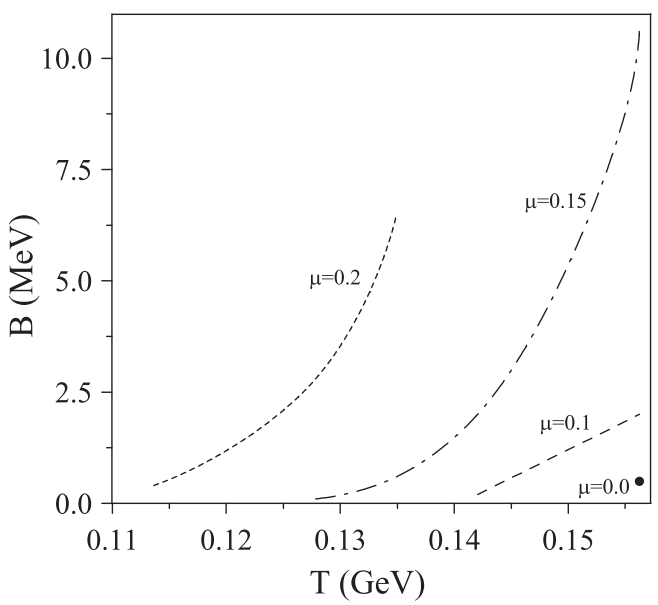

FIG. 2. Binding energy of the $(I) J^{P}=(1 / 2) 1^{+} N \Lambda_{c}$ state, as a function of the temperature $T$ for different values of the baryon chemical potential $\mu$ (in $\mathrm{GeV}$ ).

$T$ - and $\mu$-dependent parameters, we have analyzed the lowest $N \Lambda_{c}$ states, $(I) J^{P}=(1 / 2) 0^{+}$and $(I) J^{P}=(1 / 2) 1^{+}$. The $(I) J^{P}=(1 / 2) 0^{+}$channel is always repulsive. We show in Fig. 2 the binding energy of the $(I) J^{P}=$ $(1 / 2) 1^{+} N \Lambda_{c}$ state as a function of $T$ for different values of $\mu$. In all cases $B=0$ corresponds to the mass of the corresponding threshold, i.e., $M_{N}(T, \mu)+M_{\Lambda_{c}}(T, \mu)$. It can be seen how the $\Lambda_{c}$ starts to be bound as $T$ and $\mu$ increase. In fact, for $\mu=0$ there is only a tiny binding for the largest temperature considered. The values of the binding energies are small despite the large decrease in the masses of the nucleon and the $\Lambda_{c}$. Note that the largest binding obtained is of the order of $10 \mathrm{MeV}$, a value comparable to the binding energies of charmed hypernuclei in Refs. [19,21,29] and also with the $20 \mathrm{MeV}$ mass shift obtained in Ref. [33] at the normal nuclear matter density when using the unfactorized four-quark condensate. We can identify several competing effects that add or cancel to arrive to this final value. First of all, we note that the $\Lambda_{c} \leftrightarrow$ $\Sigma_{c}$ conversion is less important than in the similar system in strange sector, mainly due to the larger vacuum mass difference, namely $168 \mathrm{MeV}$ as compared to $73 \mathrm{MeV}$ in the strange sector. Besides, it comes reduced as compared to the strange sector due to the absence of strange meson exchanges [72], giving rise to a smaller $N \Lambda_{c} \leftrightarrow N \Sigma_{c}$ transition potential. Finally, the $\Lambda_{c} \leftrightarrow \Sigma_{c}$ conversion comes also suppressed when increasing $T$ and/or $\mu$, due to a larger mass difference, as seen in Fig. 1. However, the increase of the interacting potential due to the decrease of $m_{\sigma}$ is enough to give binding despite the smaller threshold mass, increasing the kinetic energy contribution.

We have performed the same analysis for $\Lambda_{c} \Lambda_{c}$ system. We show in Fig. 3 the results for the lowest channel, $(I) J^{P}=(0) 0^{+}$. This system was studied in vacuum in Ref. [46], concluding the nonexistence of a charmed $H$-like dibaryon, although it may appear as a resonance above the 


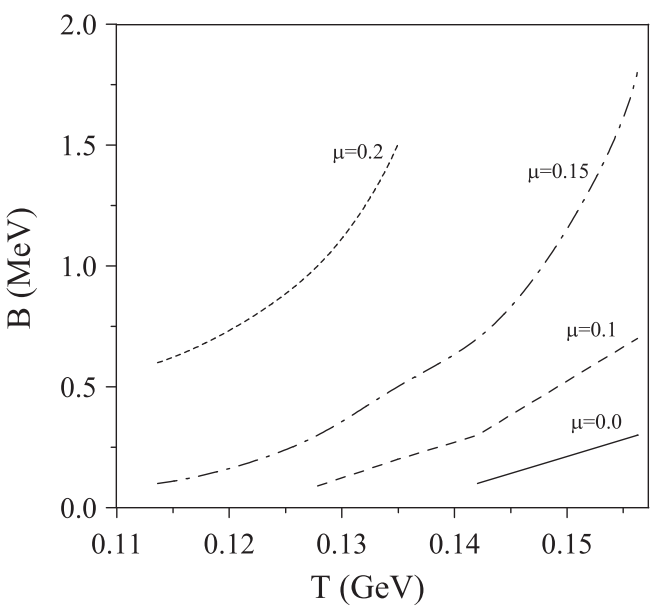

FIG. 3. Binding energy of the $(I) J^{P}=(0) 0^{+} \Lambda_{c} \Lambda_{c}$ system, as a function of the temperature $T$ for different values of the baryon chemical potential $\mu$ (in $\mathrm{GeV}$ ).

$\Lambda_{c} \Lambda_{c}$ threshold. It is important to note that the $\Lambda_{c} \Lambda_{c}$ system is decoupled from the closest two-baryon threshold, the $N \Xi_{c c}$ state, that in the case of the strange $H$ dibaryon becomes relevant for its possible bound or resonant character [73]. The binding of the $(I) J^{P}=(0) 0^{+}$state would then require a stronger attraction in the diagonal channels or a stronger coupling to the heavier $\Sigma_{c} \Sigma_{c}$ state. However, the mass difference between the two coupled channels in the $(I) J^{P}=(0) 0^{+}$partial wave, namely the $\Lambda_{c} \Lambda_{c}$ and $\Sigma_{c} \Sigma_{c}$, are much larger than in the counterpart strange sector, and increase both with $T$ and $\mu$, making the coupled-channel effect less important. Let us note that in the strange sector one has $M(N \Xi)-M(\Lambda \Lambda)=25 \mathrm{MeV}$ and $M(\Sigma \Sigma)-M(\Lambda \Lambda)=154 \mathrm{MeV}$. In the charm sector, the closest channel coupling to $\Lambda_{c} \Lambda_{c}$ in the $(I) J^{P}=(0) 0^{+}$ state is $\Sigma_{c} \Sigma_{c}$, which in vacuum is $338 \mathrm{MeV}$ above.

Thus, when increasing the temperature and/or the chemical potential, the $\Lambda_{c} \Lambda_{c}$ and $\Sigma_{c} \Sigma_{c}$ thresholds are separated and, therefore, not much further binding can be expected from coupled-channel effects. Furthermore, the decrease of $\Lambda_{c}$ mass increases the repulsion due to an increase in the kinetic energy and these two effects can only be compensated by the increase of the interacting potential. The net effect is that the in-medium $\Lambda_{c} \Lambda_{c}$ binding is much smaller than the in-medium $\Lambda_{c} N$ binding.

\section{SUMMARY}

In brief, we have studied the effect of temperature $T$ and baryon chemical potential $\mu$ on the masses of the $\Lambda_{c}, \Sigma_{c}$ and $\Sigma_{c}^{*}$ charmed baryons and on the $\Lambda_{c} N$ and $\Lambda_{c} \Lambda_{c}$ interactions. We have used a chiral constituent quark model, in which the parameters are taken to be $T$ - and $\mu$-dependent as predicted by the Nambu-Jona-Lasinio model. We have found that while the mass of the $\Lambda_{c}$ baryon decreases monotonically in medium, the masses of the $\Sigma_{c}$ and $\Sigma_{c}^{*}$ have a nonmonotonic behavior. We have shown that the behavior of the in-medium masses of those baryons can be understood in terms of a simple group theory analysis, which allows us to disentangle the dynamics of the different heavy-light and light-light two-quark subsystems composing the baryons. Thus, these systems offer a unique laboratory for learning on temperature and density effects on the phenomenon of dynamical chiral symmetry breaking. We have compared our results with others in the literature using different models, relativistic mean field models for nuclear matter and QCD sum rules.

Regarding the $\Lambda_{c} N$ and $\Lambda_{c} \Lambda_{c}$ in-medium interactions, we found that there is a delicate balance involving a stronger interaction due to the decrease of the mass of $\sigma$, a suppression of coupled-channel effects and lighter thresholds, leading to an overall effective attraction. We have found an in-medium $\Lambda_{c} N$ binding energy of the order of $10 \mathrm{MeV}$, in qualitative agreement with a calculation using QCD sum rules in which the density dependence of the unfactorized four-quark condensate is estimated from a perturbative chiral quark model. Our result clearly points to the possibility that the $\Lambda_{c}$ can be bound in a sufficiently large nucleus, as a binding energy of $10 \mathrm{MeV}$ is comparable to the binding energies found in calculations of $\Lambda_{c}$ hypernuclei using relativistic mean field. For the $\Lambda_{c} \Lambda_{c}$ system, we found a shallow bound state with a binding energy of the order of $2 \mathrm{MeV}$. Such systems can in principle be formed through coalescence in the environment of a heavyion collision. The general conclusion is that the $\Lambda_{c} N$ and $\Lambda_{c} \Lambda_{c}$ systems that are not bound in vacuum, could become bound in a medium at finite temperature and finite baryon density. Our findings are relevant for ongoing heavy-ion experiments at the Relativistic Heavy-Ion Collider (RHIC) and the LHC, and for the planned experiments at FAIR and J-PARC.

As already mentioned, charmed hadrons can be produced and their interactions realistically measured in highenergy heavy-ion collisions. In addition, the future research programs at different facilities like FAIR and J-PARC are expected to improve our knowledge on the inmedium hadron-hadron interactions involving heavy flavors. While the scarce experimental information leaves room for some degree of speculation in the study of processes involving charmed hadrons, the situation can be ameliorated with the use of well constrained models based as much as possible on symmetry principles and analogies with other similar processes. The present detailed theoretical investigation of the behavior of the in-medium masses of charmed hadrons and their interactions is based on well established models. It is hoped that our work is of help toward raising the awareness of experimentalists that it is worthwhile to investigate few-baryon systems involving heavy-flavor hadrons, specifically because for some quantum numbers such states could form interesting bound states. 


\section{ACKNOWLEDGMENTS}

This work has been partially funded by Ministerio de Economía, Industria y Competitividad and EU FEDER under Contract No. FPA2016-77177 and by a bilateral agreement Universidad de Salamanca - Fundação de Amparo à Pesquisa do Estado de São Paulo FAPESP Grant No. 2017/50269-7. Partial financial support is also acknowledged from Conselho Nacional de Desenvolvimento Científico e Tecnológico- $\mathrm{CNPq}$, Grants No. 168445/2017-4 (C. E. F.), 305894/2009-9 (G. K.), 464898/2014-5(G.K) (INCT Física Nuclear e Aplicações), and Fundação de Amparo à Pesquisa do Estado de São Paulo - FAPESP, Grant No. 2013/01907-0 (G. K.).
[1] A. Esposito, A. Pilloni, and A. D. Polosa, Phys. Rep. 668, 1 (2017).

[2] R. A. Briceno et al., Chin. Phys. C 40, 042001 (2016).

[3] J.-M. Richard, Few-Body Syst. 57, 1185 (2016).

[4] H.-X. Chen, W. Chen, X. Liu, Y.-R. Liu, and S.-L. Zhu, Rep. Prog. Phys. 80, 076201 (2017).

[5] R. F. Lebed, R. E. Mitchell, and E. S. Swanson, Prog. Part. Nucl. Phys. 93, 143 (2017).

[6] A. Ali, J. Sören Lange, and S. Stone, Prog. Part. Nucl. Phys. 97, 123 (2017).

[7] F.-K. Guo, C. Hanhart, U.-G. Meißner, Q. Wang, Q. Zhao, and B.-S. Zou, Rev. Mod. Phys. 90, 015004 (2018).

[8] C. Patrignani et al. (Particle Data Group Collaboration), Chin. Phys. C 40, 100001 (2016).

[9] T. Matsui and H. Satz, Phys. Lett. B 178, 416 (1986).

[10] A. Hosaka, T. Hyodo, K. Sudoh, Y. Yamaguchi, and S. Yasui, Prog. Part. Nucl. Phys. 96, 88 (2017).

[11] G. Krein, A. W. Thomas, and K. Tsushima, Prog. Part. Nucl. Phys. 100, 161 (2018).

[12] S. Ogilvy (LHCb Collaboration), in Proceedings of the 7th International Workshop on Charm Physics, edited by A. A. Petrov (SLAC, Detroit, 2015).

[13] R. Aaij et al. (LHCb Collaboration), Phys. Rev. Lett. 118, 182001 (2017).

[14] S. Cho et al. (ExHIC Collaboration), Prog. Part. Nucl. Phys. 95, 279 (2017).

[15] H. Noumi, JPS Conf. Proc. 17, 111003 (2017).

[16] H. Fujioka et al., arXiv:1706.07916.

[17] U. Wiedner (PिANDA Collaboration), Prog. Part. Nucl. Phys. 66, 477 (2011).

[18] C. Höhne et al., Lect. Notes Phys. 814, 849 (2011).

[19] K. Tsushima and F. C. Khanna, Phys. Rev. C 67, 015211 (2003).

[20] C.-H. Cai, L. Li, Y.-H. Tan, and P.-Z. Ning, Europhys. Lett. 64, 448 (2003).

[21] K. Tsushima and F. C. Khanna, J. Phys. G 30, 1765 (2004).

[22] Y.-H. Tan and P.-Z. Ning, Europhys. Lett. 67, 355 (2004).

[23] V. B. Kopeliovich and A. M. Shunderuk, Eur. Phys. J. A 33, 277 (2007).

[24] Z.-G. Wang, Eur. Phys. J. C 71, 1816 (2011).

[25] Z.-G. Wang, Phys. Rev. C 85, 045204 (2012).

[26] Y.-R. Liu and M. Oka, Phys. Rev. D 85, 014015 (2012).

[27] M. Oka, Nucl. Phys. A914, 447 (2013).

[28] H. Huang, J. Ping, and F. Wang, Phys. Rev. C 87, 034002 (2013).
[29] H. Garcilazo, A. Valcarce, and T. F. Caramés, Phys. Rev. C 92, 024006 (2015).

[30] S. Maeda, M. Oka, A. Yokota, E. Hiyama, and Y.-R. Liu, Prog. Theor. Exp. Phys. 023D02 (2016).

[31] J. Haidenbauer and G. Krein, Eur. Phys. J. A 54, 199 (2018).

[32] K. Azizi, N. Er, and H. Sundu, Nucl. Phys. A960, 147 (2017).

[33] K. Ohtani, K. J. Araki, and M. Oka, Phys. Rev. C 96, 055208 (2017).

[34] R. Chen, A. Hosaka, and X. Liu, Phys. Rev. D 96, 116012 (2017).

[35] C. E. Fontoura, F. Krmpotić, A. P. Galeão, C. De Conti, and G. Krein, J. Phys. G 45, 015101 (2018).

[36] T. Miyamoto et al. (HAL QCD Collaboration), Nucl. Phys. A971, 113 (2018).

[37] T. Miyamoto et al. (HAL QCD Collaboration), PoS Hadron2017, 146 (2018).

[38] K. Azizi and N. Er, Nucl. Phys. A970, 422 (2018).

[39] L. Meng, N. Li, and S.-L. Zhu, Eur. Phys. J. A 54, 143 (2018).

[40] S. Maeda, M. Oka, and Y.-R. Liu, Phys. Rev. C 98, 035203 (2018).

[41] W. Meguro, Y.-R. Liu, and M. Oka, Phys. Lett. B 704, 547 (2011).

[42] N. Lee, Z.-G. Luo, X.-L. Chen, and S.-L. Zhu, Phys. Rev. D 84, 014031 (2011).

[43] N. Li and S.-L. Zhu, Phys. Rev. D 86, 014020 (2012).

[44] L. Zhao, N. Li, S.-L. Zhu, and B.-S. Zou, Phys. Rev. D 87, 054034 (2013).

[45] H. Huang, J. Ping, and F. Wang, Phys. Rev. C 89, 035201 (2014).

[46] T. F. Caramés and A. Valcarce, Phys. Rev. D 92, 034015 (2015).

[47] L. Meng, N. Li, and S.-L. Zhu, Phys. Rev. D 95, 114019 (2017).

[48] R. Chen, F. L. Wang, A. Hosaka, and X. Liu, Phys. Rev. D 97, 114011 (2018).

[49] B. Yang, L. Meng, and S.-L. Zhu, arXiv:1810.03332.

[50] K. Saito, K. Tsushima, and A. W. Thomas, Prog. Part. Nucl. Phys. 58, 1 (2007).

[51] K. Hagino and J. M. Yao, International Review of Nuclear Physics 10, 263 (2016).

[52] T. D. Cohen, R. J. Furnstahl, D. K. Griegel, and X.-m. Jin, Prog. Part. Nucl. Phys. 35, 221 (1995). 
[53] A. Valcarce, H. Garcilazo, F. Fernández, and P. González, Rep. Prog. Phys. 68, 965 (2005).

[54] J. Vijande, F. Fernández, and A. Valcarce, J. Phys. G 31, 481 (2005).

[55] A. Valcarce, H. Garcilazo, and J. Vijande, Phys. Rev. C 72, 025206 (2005).

[56] A. Valcarce, H. Garcilazo, and J. Vijande, Eur. Phys. J. A 37, 217 (2008).

[57] Y. Nambu and G. Jona-Lasinio, Phys. Rev. 122, 345 (1961).

[58] Y. Nambu and G. Jona-Lasinio, Phys. Rev. 124, 246 (1961).

[59] T. F. Caramés, C. E. Fontoura, G. Krein, K. Tsushima, J. Vijande, and A. Valcarce, Phys. Rev. D 94, 034009 (2016).

[60] A. de Rújula, H. Georgi, and S. L. Glashow, Phys. Rev. D 12, 147 (1975).

[61] G. S. Bali, Phys. Rep. 343, 1 (2001).

[62] T. Eguchi, Phys. Rev. D 14, 2755 (1976).

[63] S. P. Klevansky, Rev. Mod. Phys. 64, 649 (1992).
[64] U. Vogl and W. Weise, Prog. Part. Nucl. Phys. 27, 195 (1991).

[65] T. Hatsuda and T. Kunihiro, Phys. Rep. 247, 221 (1994).

[66] M. Buballa, Phys. Rep. 407, 205 (2005).

[67] P. Gerber and H. Leutwyler, Nucl. Phys. B321, 387 (1989).

[68] E. G. Drukarev and E. M. Levin, Prog. Part. Nucl. Phys. 27, 77 (1991).

[69] A. Valcarce, H. Garcilazo, and J. Vijande, Phys. Lett. B 733, 288 (2014).

[70] S. Cho, T. Furumoto, T. Hyodo, D. Jido et al. (ExHIC Collaboration), Phys. Rev. Lett. 106, 212001 (2011).

[71] H. Garcilazo, J. Phys. G 13, L63 (1987).

[72] H. Bandō and S. Nagata, Prog. Theor. Phys. 69, 557 (1983).

[73] T. Inoue, S. Aoki, T. Doi, T. Hatsuda, Y. Ikeda, N. Ishii, K. Murano, H. Nemura, and K. Sasaki (HAL QCD Collaboration), Nucl. Phys. A881, 28 (2012). 